\title{
Cintigrafía de glóbulos rojos marcados con tecnecio en el diagnóstico diferencial entre hemangiomas periféricos y otras masas blandas
}

\author{
Claudio Opazo R. ; Patricio González E. ;; Julia Oroz M.; Sergio Silva V.; \\ Paulina Grandi P.2; Nancy Garcés V. ${ }^{3}$
}

\author{
Differential diagnosis of hemangiomas and other soft-tissue \\ masses by technetium-labeled red blood cell scintigraphy
}

\begin{abstract}
The usefulness $99 \mathrm{~m}^{-} \mathrm{Tc}$ iabeled red blood cell pool scinligrophy to differentiale between peripheral hemangiomas and non hemangiomotous lessions was evoluated in thifly six potienis in whom hemangioma or other lession were suspected. Flanar scans were assessed. Uptake from lessions was appraised by visual inspection, and its injensity was classified in four degrees. An index count indexi was also calculated from counts recorded on the area of the lession divided by those obtained from a reference area. Once delinite -clinical or pathological-diagnosis were obtained, patienls were seporaled in two groups: hemangiomas iHI. $n: 20$, and non hemangiornas $[\mathrm{NHI}, \mathrm{n}: 10$. The overage uplake inlensity of lesions in group $\mathrm{H}$ was $2.9 \pm 1.1$ in compousson to $1.5 \pm 1.1$ in NH patients. The corresponding overage count index was $2.2 \pm 1.1$ in group $\mathrm{H}$ cases and $1.2 \pm 0.3$ in $\mathrm{NH}$ group. Bcth criterio were significantly different $(p<0.0013)$ among both groups of palients and they seem to be useful to differentiate hemangiomas from other soft-tissue lessions.
\end{abstract}

(Koy words: hernangioma, scintigraphy, radionuclide -labeled red blcad cell, vascuiar malformation.)

Las lesiones vasculares cutáneas y en particular los hemangiomas, son un problema frecuente en pediatría. De acuerdo a la clasificación propuesı por Mulliken y Glowacki ${ }^{1}$ pueden ser divididas en dos grupos: hemangiomas y malformaciones vasculares. Esta diferenciación está basada en características endoteliales y tiene importantes implicancias pronósticas y terapéuticas.

Los hemangiomas son tumores benignos del endotelio vascular, caracterizados por evolucionar en una fase proliferativa, una estacionaria y una involutiva. Crecen rápidamente durante la lactancia, se estabilizan e involucionan con lentitud. Sus principales tipos son los hemangiomas cavernoso, tuberoso y mixto. Son los tumores más frecuentes del periodo de lacuncia (10 a $12 \%$ de todos los de nirtos menores de un año).

1. Centro de Medicina Nuclear, Hospital Clínico de la Universidad de Chilc.

2. Servicio de Dermalología, Hospital Roberto del Río.

3. Tecnólogo Médico. Centro de Mcdicina Auclear, Hospital Clínico de la Universidad de Chile.
Las malformaciones vasculares son, en cambio, anomalias del desarrollo, cuyo crecimiento está en relación con el del cuerpo, por lo que no involucionan espontáneamente. Pueden ser de origen capilar (como la mancha en vino de Oporto $\mathrm{y}$ cl sindrome de Klippel Trenaunay), o bien linfático (como el linfangioma y de grandes vasos como las fístulas artcriovenosas congénitas). Debido a su pronóstico, el tratamiento de los hemangiomas es, por lo general, conservador e incluye desde la simple observación hasta tratamientos con corticoides u otros medios ${ }^{2}$.

La distinciön entre estos dos lipos de lesiones se realiza habitualmente en base a los signos clínicos $^{3}$, sin embargo, en algunas ocasiones el diagnostico diferencial puede ser difícil, en especial cuando se trata de hemangiomas en las primeras fases de su evolución ${ }^{4}$, necesitando exámenes complementarios. La resonancia nuclear magnética parece ser un método útil de diferenciación ${ }^{5-7}$ y se prefiere a la tomografía axial computadorizada, pues ćsta requiere emplear medio de contraste e imágenes dinámicas ${ }^{4,8,9}$. 
También se ha utilizado la ecografía, pero por este método no se obtienen imágenes específicas $^{10}$ y sus resultados son muy dependientes del operador. La angiografía es un método invasivo que utiliza medio de contraste y que se emplea principalmente antes de hacer tratamientos por embolización o cirugía ${ }^{4}$.

Buscando un método objetivo y menos invasivo se ha utilizado la cintigrafía con glóbulos rojos marcados con Tc99m (GR-Tc99m), con la que se ha demostrado adecuada sensibilidad y especificidad para diferenciar hemangiomas de malformaciones vasculare ${ }^{4}$, como también que es útil para distinguir entre lesiones cutáneas vasculares y de otro origen ${ }^{12}$. El procedimiento tiene la ventaja de permitir una exploración de cuerpo entero para detectar hemangiomas en otras localizaciones $^{13}$.

El propósito de este estudio fue evaluar la utilidad de la cintigrafía con GR-Tc99 m para diferenciar hemangiomas de malformaciones vasculares y de lesiones de otro origen -como tumores-, empleando criterios cuantitativos y semicuantítativos.

\section{Pacientes y Méttodos}

Entre octubre de 1991 y febrero de 1993,36 pacientes (23 mujeres), cuya edad promedio era 3 años, variación un mes a 9 años, fueron referidos por médicos dermatólogos con el diagnóstico presuntivo de hemangioma, malforma. ción vascular u orro tumor de tejidos blandos. A todos ellos se les realizó cintigrafía con GR-Tc99m, planar, en gamacámara STAR/CAM A/CT 3200 con colimador de alta resoluciốn, obteniéndose imágencs de cuerpo entero, antero. posteriores-posteroanteriores, así como de la zona de la lesión en diversas proyecciones. Los globulos rojos se marcaron en vivo con $\mathrm{Tc} 99 \mathrm{~m}$-clonuro estanoso y perclorato dos horas antes del esuidio. La dosis de marcador se calculó en proporción a la superficie corporal, comando come referencia una de $30 \mathrm{mCi}$ para el adulto.

La evaluación de las imágenes se efectuó medjante inspección visual, midiendo el tamaño de las lesiones y clasificando la intensidad de captación que mostraban las lesiones en una escala de 1 a 4 (correspondiendo el grado 1 a una intensidad menor que la de vasculatura; grado 2 igual a vasculatura; grado 3 mayor a vasculatura; grado 4 muy intenso). Además se calculo, mediante un computador, un indice de actividad, para lo cual se compararon las cuentas de una región de interés, dibujada sobre la lesión, con las obtenidas en un área anatómicamente comparable, habitualmente la contralateral, donde no había lesión.

Los diagnósticos definitivos se obuvieron por biopsia (10 casos) y por evolución clínica (26 casos), después de lo cual los pacientes fueron separados en dos grupos: hemangiomas y otro lipo de lesiones que no eran hemangiomas.
Catorce de los casos de hemangiomas eran del tipo cavernoso, 11 mixtos y uno múltiple benigno. De los diez casos cuyas lesiones no eran hemangiomas, seis tenian malfonnaciones vasculares (cuatro linfangiomas y dos manchas vino Oporto); de los restanles curatro no hemangiomas, dos eran hemangiopericitomas, uno fibrosarcoma y atro correspondia a hipertofia no vascular.

Debido a las características de la muestra (diferenciadas de varianzas, tamaño y variable cualitativa en el caso de la calificación de intensidad) se utiliz6 la prueba de Wilcoxon para muestras independientes. Se contrastaron las sumas de los márgenes de los valores obtenidos entre los grupos de hernangiomas y no hemangiomas, tanto para la calificacion de intersidad como para el índice de actividad. Se consideró significativo un valor de $\mathrm{p} \leq 0,05$.

\section{Resultados}

Por lo general en los hemangiomas la captación de GR Tc99m estaba aumentada -siendo al menos igual y frecuentemente mayor a la de la vasculatura adyacente-, era focal y además bjen delimitada (figura 1). En los linfangiomas la captación fue menor que la de vasculatura (figura 2). Lo mismo se observó en las manchas en vinoOporto y en un fibrosarcoma (figura 3). Hubo dos casos de hemangiopericitomas. Uno mostró marcado contraste (intensidad 4 , índice de actividad 1,9) y el otro captación similar al tejido circundante (índice e intensidad iguales a 1).

La calificación de intensidad, cuyo promedio en los hemangiomas fue $2,9 \pm 1,0$ y en los no herangiomas $1,5 \pm 1,0$, mostró diferencias significalivas al comparar las sumas de los márgenes entre ambos grupos $(\mathrm{p}<0,0013)$. El promedio de los indices de actividad obtenidos en los hemangiomas fue $2,2 \pm 1,0$ y en los no hemangiomas $1,2 \pm 0,3$. Las sumas de los márgenes de ambos grupos también difirieron significativamente ( $\mathrm{p}<$ 0,0013 ) (figura 4).

\section{Comentario}

Los hemangiomas son frecuentes en pediatría. Tienden a involucionar espontáneamente, por lo que es importante diferenciarlos de otras causas de aumento de volumen de partes blandas cuyo pronóstico y tratamiento puede diferir notablemente. El diagnóstico se efectúa clínicamente en la mayoría de los casos, sin embargo, en ocasiones esta distinción es difícil.

Los estudios ecográficos han sido utilizados en la evaluación preliminar para determinar el 

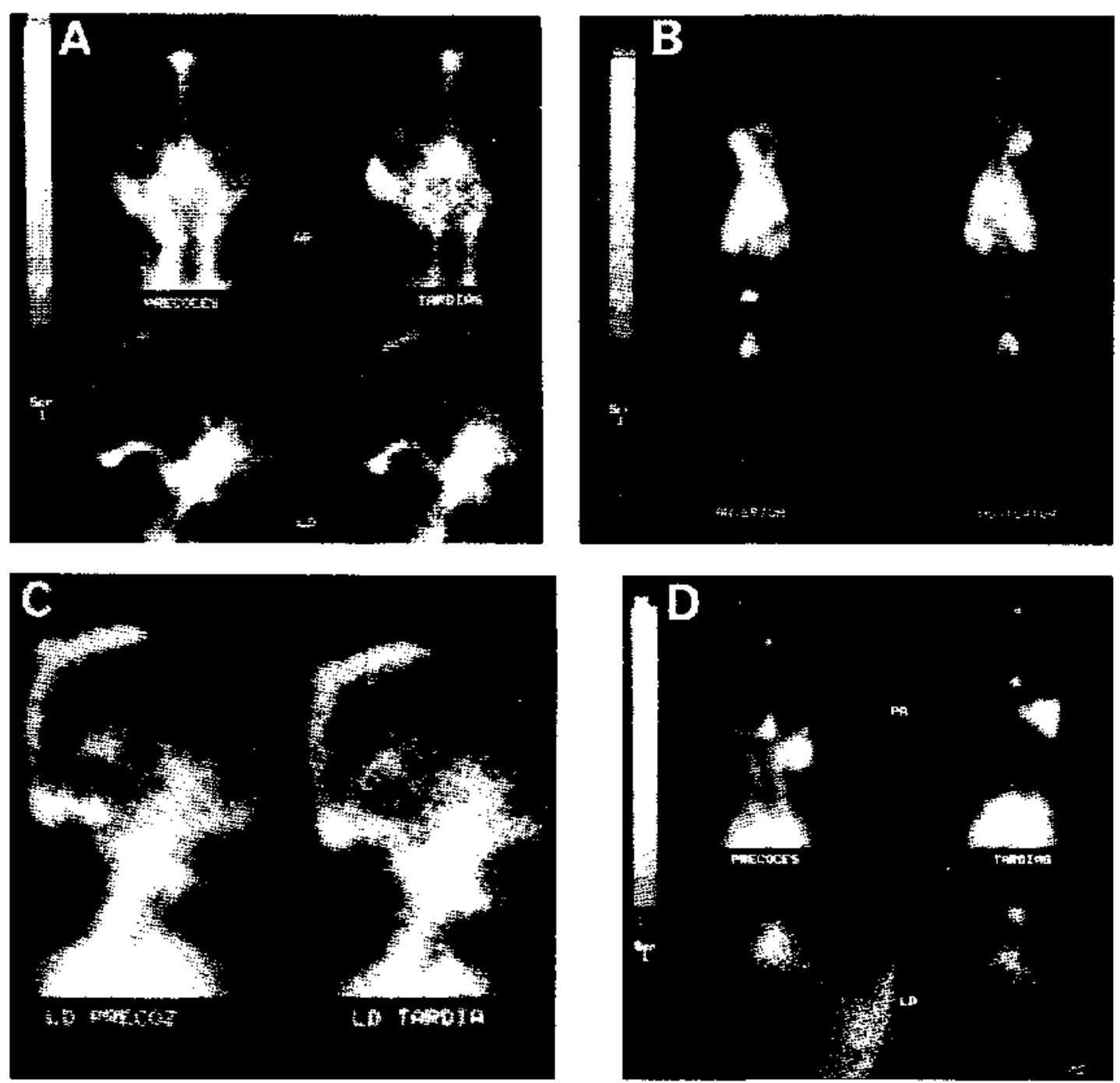

Flgura 1. Hemangioma cavemoso de la mejilla derecha en una nin̄a dę dos ăos. Imágenes obtenidas 5 minutos después de la inyección: área focal de intensa captación del trazador en esa zona. En los controles tardíos, dos boras después, se aprecia refuerzo de la intensidad en la lesión, lo cual onienta a este tipo de hemangiona. b: Hemangioma caverauso que compronete la mejilla y la región cervical derechas. Imágenes de cuerpo entero en proyecciones anteroposterior y posteroanterior. Se observa claramente el área de vascularidad marcadamente aumentada en esa zona, más jntensa quc en los vasos sanguíneos cervicales. c: Hemangioma mixto en la línca media del cuero cabelludo. Proyecciones laterales derechas, precoces y tardías. d: Hemangiona mixto en región cervical derecha posterior. Imágenes precoces y tardías en proyecciones posteroanterior y lateral derecha.

tamaño y ubicación anatómica de la lesión. Ellos tienen la ventaja de ausencia de radiación ionizante, aunque no proporcionan imágenes específicas y eventualmente deben ser complementados con otros procedimientos ${ }^{10}$.

La resonancia nuclear magnética informa acerca de la extensión del compromiso en los planos tisulares y pucde discriminar entre hemangiomas y malformaciones arteriovenosas ${ }^{4}, \mathrm{La}$ tomografía axial computadorizada con medio de contraste puede diferenciar malformaciones venosas y linfáticas, requiere a menudo de estudio dinámico, lo que aumenta la magnitud de la irradiación. Las limitaciones de estos dos méto- 

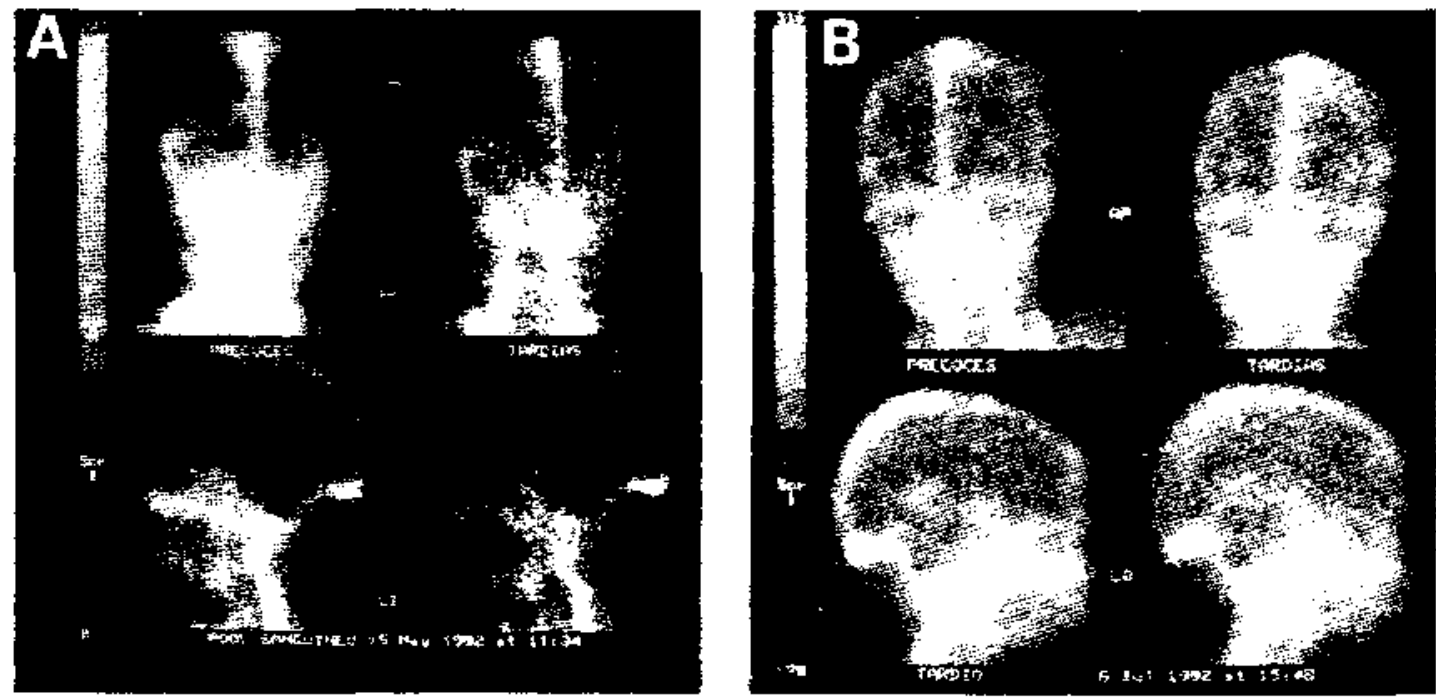

Figura 2. a: Linfangioma en una niña de tres años. Proyecciones antejoposteriores y laterales izquicrdas. En las proyeccioncs AP se observa en mejilla izquierda, en correspondencia con el aumento de volumen existente a ese nivel, una zona tenuemente contrastada, lo cual indica un tejido relativamente avascular. b: Linfangioma de mejilla dercecha, cl cual se observa en las proyecciones anteroposteriores como un área de poca vascularidad.

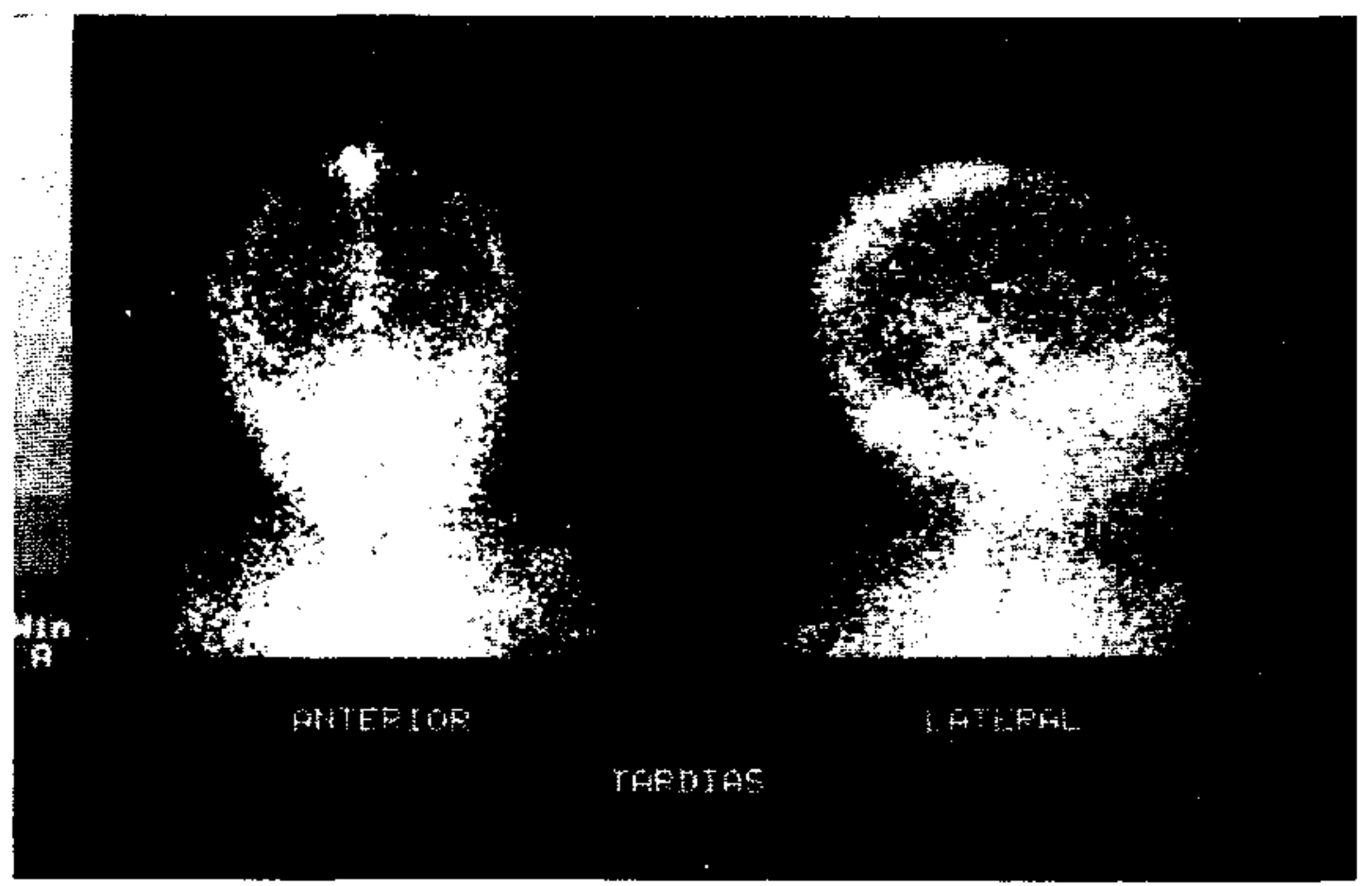

Figura 3. Fibrosarcorna parietal derecho que se observa en la proyección anteroposterior como uma zona de leve mayor acumulación del trazador, difusa, sin aspecto de hemangioma. 


\section{INDICES}

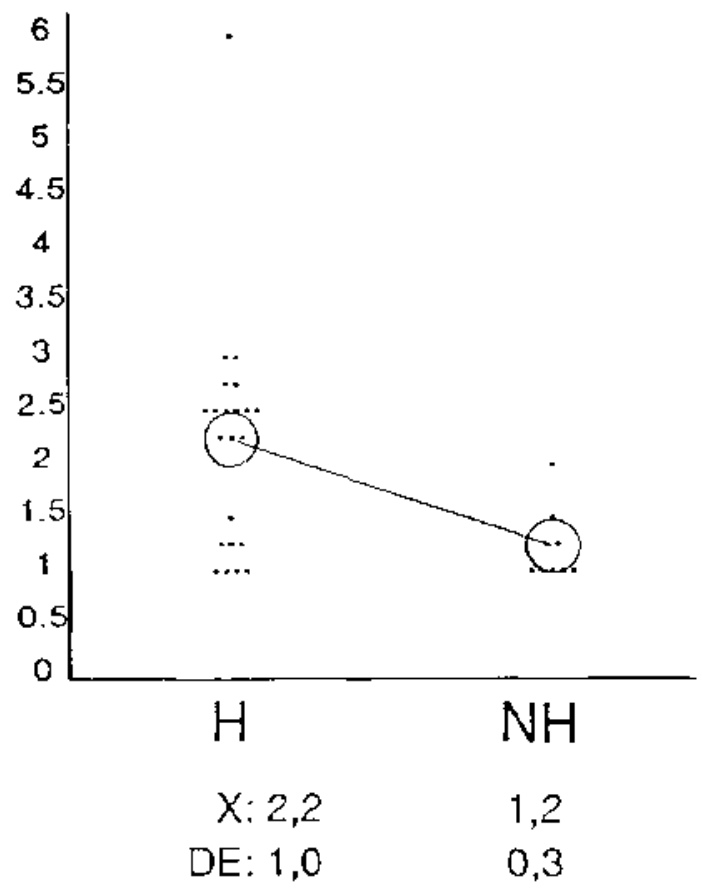

$\mathrm{H}$ : hemangiograma
CALIFICACION

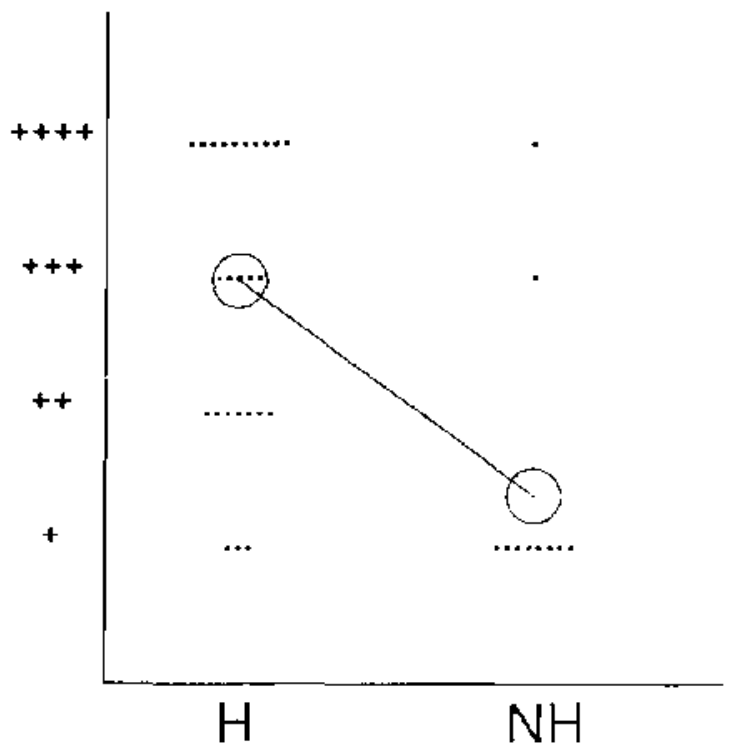

$\begin{array}{ll}2,9 & 1,5 \\ 1,1 & 1,1\end{array}$

$\mathrm{NH}$ : otras lesiones de partes blandas

Figura 4: Indices de captación y calificación de la intensidad de la captación oblenidos en hemangiomas y lesiones no hemangiơnatosas. Se indican los promedios y las desviaciones estándas.

dos es que en nuestro medio son poco accesibles $y$ tienen alto coste.

La angiografía permite una adecuada diferenciación entre hemangiomas y malformaciones vasculares ${ }^{11}$, pero su empleo es limitado por ratarse de un procedimiento invasivo. La cintigrafía con GR-Tc99m se ha usado con buenos resultados para este propósito, con la ventaja de no ser invasiva. Sloan, en 1988, estudio su utilidad para distinguir entre malformaciones vasculares y otras masas de tejidos blandos en nihos, demostrando una sensibilidad de $98 \%$ y una especificidad de $100 \% 12$. Posteriormente se ha demostrado que el estudio tenía $97 \%$ de exactitud para diferenciar entre hemangiomas y otras alteraciones vascularest.

Los resultados que hemos expuesto confurman la utilidad de la cintigrafía de pool sanguíneo para distinguir los hemangiomas de otras lesiones en base al grado de vascularidad. Sólo el hemangiopericitoma muestra captación intensa, semejante a la de los hemangiomas. La utilización de criterios semicuantitativos, como la calificación en punto de la intensidad de captación y el índice de actividad, permite disponer al médico nuclear de información objetiva para la diferenciación, ayudando al clínico en caso de dudas sobre el diagnóstico.

\section{Resumen}

Se evaluó la utilidad de la cintigrafia con glóbulos rojos marcados en el diagnóstico diferencial entre hemangiomatosis periféricas y otras lesiones no hemangiomatosas. Se estudiaron 36 pacientes con diagnóstico presuntivo de hemangionas u otras lesiones de partes blandas como malformaciones vasculares y tumores. El estudio cintigráfico se realiz $\delta$ empleando la modalidad planar y la evaluación de las lesiones se hizo mediante inspección visual, clasificando la intensidad de contraste en una escala de calificaciones de cuatro grados. Además se calculó, me- 
diante computador, un índice de actividad dividiendo las cuentas obtenidas sobre la lesión por las registradas en un área corporal sana de control. Una vez obtenidos los diagnósticos definitivos (por biopsia y evolución clínica) los casos fueron separados en hemangiomas y no hemagiomas. En el grupo de los hemangiomas la calificación promedio de la intensidad de la capıación fue $2,9 \pm 1,1$ y en el de los no hemangiomas $1,5 \pm 1,1$. El índice de actividad promedio en los casos de hemangiomas fue $2,2 \pm 1,1$ y en los no hemangiomas $1,2 \pm 0,3$. Las diferencias fucron estadísticamente significalivas entre ambos grupos para los dos criterios, que pueden, en conclusión, considerarse útiles para diferenciar hemangiomas de otras lesiones.

(Palabras clave: hemangioma, cintigrafía, Tc99m, glóbulos rojos marcados, malformación vascular.)

\section{Referencias}

1. Mulliken JB, Glowacki $J$ : Hemangiomas and vascular matfomations in infants and children: A classification based on endothelial characteristics. Plast Reconstr Surg 1982; 69: 412-420.

2. Casillo P, Corradini $E$, Oroz $J$ : Hemangiomas y malformaciones vasculares. Seminario de Dematología
Pediálrica. Servicio de Dennatología. Hospital Clínico de la U. de Chile 1992.

3. Mulliken $J B$, Young $A E$ : Vascular Birthmarks: Hernangiomas and malformations. Philadelphia: Saunders, 1988.

4. Barton D, Müller J: Distinguishing sofi-tissue herrangiomas from vascular malformations using technetiumlabeled red bood cell scintigraphy. Plast Reconstr Surg $1992 ; 89: 46-55$.

5. Kransdor $M$, Jelinek $J$ : Soft-tissue masses: Diagnosis using MR imaging. AJR 1989; 153: 541.547.

6. Buetow $P$, Kransdorf $M$ : Radiologic apperance of intramuscular hemangioma with emphasis on MR imaging. AJR 1990; 154: 563-567.

7. Yuh W, Kathol $M$ : Hemangiomas of skeletal muscle: MR findings in five patients. AJR 1987; 149: 765-768.

8. Tehranzadeh J, Mnaymneh W: Comparison of CT and MR imaging in musculoskeletal neoplasm. J Comput Assist Tomogr 1989; 13: 466-471.

9. Levine $E$, Wetzel $L$, Neff $J$; MR imaging and C' ${ }^{\circ}$ of extrahepatic cavernous hemangiomas. AJR 1989; 147 ; 1299-1303.

10. Bradiey $M$. Stewart $I$ : The role of ultrasound and $99 \mathrm{~m}$ T $\mathrm{CRBC}$ scintigraphy in the diagnosis of the salivary gland haemangioma. Br J Oral Maxillofac Surg 1991; 29: 164-166.

11. Burrows $P$. Mulliken $J$ : Childhood hemangionas and vascular malformations: angiographic differentiation. AJR 1983; 141: 483.488 .

12. Sloan GM, Bolion $L$ : Radionuclide labeled bood cell imaging of vasculas malformations in children. Ann Plast Surg 1988; 21: 236-241.

13. Stanley $P$, Geer $G$ : Infantile hepatic hemangiomas. Cancer 1989; 64: 936-949. 